\title{
La psicopedagogía desde el enfoque de género para la convivencia social
}

Psychopedagogy from a gender perspective for social coexistence

\author{
Graciela Hernestina Zambrano-Pincay \\ grace22zambrano@gmail.com \\ Universidad Técnica de Manabí, Portoviejo \\ Ecuador \\ https://orcid.org/0000-0003-2396-3451 \\ María Jesús Viton-de-Antonio \\ mariajesus.viton@uam.es \\ Universidad Autónoma de Madrid, Madrid \\ Ecuador \\ https://orcid.org/0000-0002-7006-8671 \\ Patricio Yosué Vallejo-Pilligua \\ patricio.vallejo@utm.edu.ec \\ Pontificia Universidad Católica del Ecuador, Portoviejo \\ Ecuador \\ https://orcid.org/0000-0001-9039-7061 \\ Patricio Alfredo Vallejo-Valdivieso \\ patricio2871@yahoo.es \\ Universidad Técnica de Manabí, Portoviejo \\ Ecuador \\ https://orcid.org/0000-0003-3248-7864
}

Recibido: 01 de mayo de 2021

Aprobado: 01 de agosto de 2021 


\title{
RESUMEN
}

Se tuvo por objetivo determinar la eficacia de un programa formativo en psicopedagogía desde el enfoque de género para la convivencia social dirigido a docentes, padres, madres, representantes, para el acompañamiento de infantes entre los 3 a 5 años en ambientes de aprendizaje de la ciudad de Portoviejo, provincia de Manabí - Ecuador. Se planteó un tipo de investigación explicativa con diseño cuasi experimental de pre y pos test con un solo grupo de trabajo o muestra poblacional de 84 personas. Se aplicó la prueba de T de Student. Al existir significancia bilateral de 0,046 para el momento de postest, se considera que el programa formativo aplicado surtió efecto en la muestra poblacional. En el campo de la lingüística, es necesario enseñar desde los primeros años de formación académica, el discernimiento de signos y códigos no feministas ocultos en la música, redes sociales.

Descriptores: Educación sexual; educación a la vida familiar; educación de la primera infancia. (Palabras tomadas del Tesauro UNESCO).

\begin{abstract}
The objective was to determine the effectiveness of a training program in psychopedagogy from the gender perspective for social coexistence aimed at teachers, parents, representatives, for the accompaniment of infants between 3 to 5 years old in learning environments of the city from Portoviejo, Manabí province - Ecuador. A type of explanatory research was proposed with a quasi-experimental pre and post-test design with a single work group or population sample of 84 people. Student's t test was applied. As there is a bilateral significance of 0.046 for the post-test moment, it is considered that the applied training program had an effect on the population sample. In the field of linguistics, it is necessary to teach from the first years of academic training, the discernment of non-feminist signs and codes hidden in music, social networks.
\end{abstract}

Descriptors: Sexuality education; population education; early childhood education. (Words taken from the UNESCO Thesaurus). 


\section{INTRODUCCIÓN}

La convivencia social en la actualidad se ve convulsionada por procesos de aceptación de nuevos roles, responsabilidades, estereotipos, en donde el modelo hombre - mujer del enfoque patriarcal, proyecta transformación por un feminismo que procura igualdad, equidad, de la mujer en tomar liderazgo de sus acciones bajo el principio de autonomía para ejercer funciones reservadas cotidianamente al varón, sin que esto en el fondo represente la pérdida de la esencia femenina.

Lo planteado, además, debe transitar en medio de un convulsionado movimiento mundial basado en el tema de identidad de género, el cual, no debe confundirse con el feminismo en su esencia, aunque existan movimientos radicales que toman el feminismo como vertiente filosófica para sustentar su visión antropológica de actuar desde el sentimiento interno como condicionante para pretender actuar desde una conducta no alineada al sexo biológico de nacimiento. En este punto, se puede destacar la existencia de una sociedad movida por el machismo, feminismo, y lo denominado como binario; cada uno representa un estereotipo de actuación social.

En este punto la escuela podría constituirse en una pequeña aldea en donde se encontrarán los tres movimientos, perspectivas, visiones, enfoques, mencionados anteriormente; siendo aquí necesario reflexionar sobre ¿si se aceptan a todos o se generan nuevos excluidos del sistema educativo? Siendo recurrente aceptarlos con la finalidad de promover una educación basada en la convivencia en alteridad social, esto implica reconocer al otro sin necesariamente ser el otro. Esto conlleva a entender a la otra persona desde la alteridad "como el actuar responsablemente frente al otro, y debo protegerlo contra todo tipo de exclusión y violencia" (Zanon, 2019, p. 76), incluyendo en este caso, la discriminación o violencia de género, siendo considerable y necesario formar para contar con una ciudadanía para el respeto mutuo a partir de las diversidades ideológicas, religiosas, políticas, en las cuales se dinamiza el ser humano. 
Es aquí donde la diversidad debe ser percibida como una oportunidad para crecer como personas en contexto del auto respeto y respeto del otro, enseñando a no etiquetar al otro por verse diferente a mí, e incluso que, si tomo acciones que naturalmente la sociedad le ha designado como normal al otro, a mi no me hace diferente o extraño por cuanto debo tener la estima positiva de quien soy realmente. Siendo en este punto donde los docentes a partir de la psicopedagogía deben aplicar estrategias en el ámbito de aprendizaje con la intención de promover la generación o construcción entre pares de una personalidad sana, asertiva, desde una concepción emocional - conductual, en relación de estimular adecuadamente

El sistema límbico conjunto anatómico conocida también como red de neuronas distribuida por el cerebro mesclado entra algunas estructuras diferentes, como el fondo de saco, el hipocampo, la circunvolución cingulada, la amígdala, la circunvolución del hipocampo y parte del tálamo. y el encéfalo que están especialmente conectadas entre sí y cuya función tiene que ver con la aparición de los estados emocionales y conductuales del ser humano (Lozano-Chaguay et al. 2020).

A partir de lo planteado, es recurrente fomentar una educación en alteridad social para poder comprender que, aunque existen diferencias biológicas y de comportamientos naturales a cada uno, se puede convivir desde la aceptación del otro sin discriminación de ningún tipo (Pérez, 2015), e incluso contrarrestar el egocentrismo (Ortega et al. 2014), el cual termina por afectar a la persona en su esencia, bien sea que esta sea varón o hembra.

Visión promovida por (Marañón, 2017), al incentivar un feminismo que eduque para formar personas seguras de sí mismas, respetuosas, sin tener en cuenta el sexo, para lo cual además propone formar para sumar un cambio de imagen, por ejemplo que el hombre pueda estar en el hogar atendiendo los niños, sin que esto represente una 
pérdida de su identidad o hombría, evitándose en este punto el equitamiento emocional negativo o errado, el cual suele constituirse en bullying dañando la memoria emocional de la persona.

En razón de lo expuesto, la investigación tiene por objetivo determinar la eficacia de un programa formativo en psicopedagogía desde el enfoque de género para la convivencia social dirigido a docentes, padres, madres, representantes, para el acompañamiento de infantes entre los 3 a 5 años en ambientes de aprendizaje de la ciudad de Portoviejo, provincia de Manabí - Ecuador.

\section{METODOLOGÍA DE INVESTIGACIÓN}

La investigación se desarrolló desde un proceso metodológico en razón de lograr el objetivo planteado, para lo cual, se planteó un tipo de investigación explicativa con diseño cuasi experimental de pre y pos test con un solo grupo de trabajo o muestra poblacional, con la finalidad de impartir un programa formativo en psicopedagogía desde el enfoque de género para la convivencia social dirigido a docentes, padres, madres, representantes, para el acompañamiento de infantes entre los 3 a 5 años en ambientes de aprendizaje de la ciudad de Portoviejo, provincia de Manabí - Ecuador.

La población que participó en el programa formativo y a la cual se le aplicó el pre y postest, estuvo conformada por 34 docentes, 23 padres y 27 madres que hacen vida en instituciones privadas donde se forman infantes entre los 3 a 5 años en ambientes de aprendizaje de la ciudad de Portoviejo, provincia de Manabí - Ecuador, por consiguiente, se trabajó con una totalidad de 84 personas en el programa formativo, siendo esta la muestra de la investigación. 


\section{Tabla 1.}

Población y muestra de la investigación.

\begin{tabular}{lc}
\hline \multicolumn{1}{c}{ Perfil poblacional } & Cantidad \\
\hline Docentes de sexo femenino & 23 \\
Docentes de sexo masculino & 11 \\
Madres representantes & 27 \\
Padres representantes & 23 \\
\hline Total & 84 \\
\hline
\end{tabular}

Elaboración: Los autores.

Como técnica de recopilación de datos se empleó la prueba y como instrumento un cuestionario tipo prueba con alternativas selectivas, siendo empleado para el momento de pre y postest de la investigación, el cual estuvo conformado por 17 ítems de alternativas de respuestas selectivas. Siendo validado en contenido por el juicio de 3 expertos y cálculo de fiabilidad por Alfa de Cronbach con resultado de 0,89 siendo considerable confiable para su aplicación.

En cuanto al tratamiento estadístico, se aplicó la prueba de $\mathrm{T}$ de Student de medias independientes con la finalidad de comparar los datos recopilados en el momento de pre y postest con lo cual, se logró medir si existió cambio estadístico o no al momento de comparar las medias independientes, para esto se tuvo apoyo del programa estadístico SPSS V25. Se aplicó el programa por medio de la plataforma zoom en razón de 7 encuentros, discriminados en los siguientes temas:

a) Emmanuel Lévinas (fundamentos de alteridad)

b) Iría Marañón (importancia de educar en el feminismo)

c) María José Urruzola Zabalza (educar en sexualidad desde el feminismo)

d) Graciela Hierro (ética desde el feminismo) 
De ese modo se abordaron cuatro temáticas en razón de incentivar la formación integral en los participantes con la finalidad de generar aprendizaje desde el programa formativo, siendo posible promover la comprensión del feminismo como una opción para educar en el respeto mutuo y vida en sexualidad sana a lo largo de la vida. El programa formativo se aplicó en razón de 7 encuentros, del siguiente modo:

Encuentro 1: Charla introductoria sobre el desarrollo del programa y aplicación del pre test.

Encuentro 2: Alteridad social desde la visión de Emmanuel Lévinas.

Encuentro 3: Importancia de educar en el feminismo desde la visión de Iría Marañón

Encuentro 4: Educar en sexualidad desde el feminismo desde la visión de María José Urruzola Zabalza.

Encuentro 5: Ética desde el feminismo desde la visión de Graciela Hierro.

Encuentro 6: Dialogo sobre los temas abordados.

Encuentro 7: Charla de cierre y aplicación del postest.

\section{RESULTADO}

Una vez aplicado el instrumento tipo prueba selectiva tanto para el momento pre y postest, el cual estuvo conformado por 17 ítems de alternativas de respuestas selectivas, se procedió a introducir las calificaciones obtenidas en correspondencia para el momento pre y momento postest en el programa estadístico SPSS V25, y se aplicó la Prueba T de Student de medias independientes, siendo reflejado el resultado en la tabla 1. 


\section{CIENCIAMATRIA}

Revista Interdisciplinaria de Humanidades, Educación, Ciencia y Tecnología

Año VII. Vol. VII. N¹. Edición Especial. 2021

Hecho el depósito de ley: pp201602FA4721

ISSN-L: 2542-3029; ISSN: 2610-802X

Universidad Nacional Experimental Francisco de Miranda (UNEFM). Santa Ana de Coro. Venezuela

Graciela Hernestina Zambrano-Pincay; María Jesús Viton-de-Antonio; Patricio Yosué Vallejo Pilligua;

Patricio Alfredo Vallejo Valdivieso

Tabla 1.

Prueba T de Student de medias independientes para programa formativo.

\begin{tabular}{lrrrrrr}
\multicolumn{7}{c}{ Prueba para una muestra } \\
Valor de prueba = 5
\end{tabular}

Al existir significancia bilateral de 0,046 para el momento de postest, se considera que existió cambio estadístico en comparación con el pretest, lo cual corresponde que el programa formativo aplicado surtió efecto en la muestra poblacional, procediéndose a aceptar la hipótesis afirmativa.

\section{Prueba de hipótesis}

H1: El programa formativo en psicopedagogía desde el enfoque de género para la convivencia social dirigido a docentes, padres, madres, representantes, para el acompañamiento de infantes entre los 3 a 5 años en ambientes de aprendizaje de la ciudad de Portoviejo, provincia de Manabí - Ecuador, fue efectivo en la muestra poblacional.

H0: El programa formativo en psicopedagogía desde el enfoque de género para la convivencia social dirigido a docentes, padres, madres, representantes, para el acompañamiento de infantes entre los 3 a 5 años en ambientes de aprendizaje de la ciudad de Portoviejo, provincia de Manabí - Ecuador, no fue efectivo en la muestra poblacional.

Se acepta $\mathrm{H} 1$ y se rechaza $\mathrm{H} 0$. 


\section{CIENCIAMATRIA}

Revista Interdisciplinaria de Humanidades, Educación, Ciencia y Tecnología

Año VII. Vol. VII. N¹. Edición Especial. 2021

Hecho el depósito de ley: pp201602FA4721

ISSN-L: 2542-3029; ISSN: 2610-802X

Universidad Nacional Experimental Francisco de Miranda (UNEFM). Santa Ana de Coro. Venezuela

Graciela Hernestina Zambrano-Pincay; María Jesús Viton-de-Antonio; Patricio Yosué Vallejo Pilligua;

Patricio Alfredo Vallejo Valdivieso

\section{DISCUSIÓN}

Educando para ser emocionalmente sanos y libres, es un imperativo necesario en las escuelas, en este sentido, "los estereotipos destruyen nuestras capacidades, nuestra autoestima y nuestra forma de concebir el mundo" (Marañón, 2017, p. 22); el antídoto a esta realidad se constituye por educar para concebirse como parte de una ciudadanía global en la cual nos movilizamos y nuestras acciones confieren beneficios 0 consecuencias hacia el otro, todo dependerá del gesto, trato, ejercido y como es interpretado por la otra persona, siendo necesario formar para la verdadera fortaleza, que no es otra manera estar emocionalmente apto para tolerar las diferencias sin perder la identidad propia.

Siendo fundamental para lograr equilibrar la estima de la niña y del niño, a partir de promover una saludable educación en sexualidad (Urruzola-Zabalza, 2000), por cuanto esta involucra no solo el conocimiento sobre los órganos sexuales, sino, como un todo que envuelve a la persona a vivir saludablemente, es aprender a valorar al otro como persona, como ser, no como un objeto o mercancía que se usa y desecha según intereses, es enseñar a amar y para esto hace falta plantear el enfoque de alteridad para mirar al otro como igual desde una visión feminista.

En este sentido, (García-Piña, 2016), destaca que la sexualidad sigue siendo un tabú en la sociedad, especialmente cuando se trata de educar a los niños y niñas, es necesario que para tal fin, los padres en primer lugar, se encuentren formados desde una óptica clínica - científica con la finalidad de promover en sus hijos, un adecuado acompañamiento formativo, el cual, debe ser complementado en la escuela, siendo esta una de las principales responsabilidades de la psicopedagogía, por cuanto debe ubicar las estrategias adecuadas para transmitir el tema de la sexualidad, y a su vez, los padres se retroalimenten, de ese modo, se genera un espacio de estudio para constituir un equipo de trabajo para proyectar una educación afectiva - sexual en concordancia con una visión de alteridad y enfoque feminista. En complemento, es necesario que en la 


\section{CIENCIAMATRIA}

Revista Interdisciplinaria de Humanidades, Educación, Ciencia y Tecnología

Año VII. Vol. VII. N¹. Edición Especial. 2021

Hecho el depósito de ley: pp201602FA4721

ISSN-L: 2542-3029; ISSN: 2610-802X

Universidad Nacional Experimental Francisco de Miranda (UNEFM). Santa Ana de Coro. Venezuela

Graciela Hernestina Zambrano-Pincay; María Jesús Viton-de-Antonio; Patricio Yosué Vallejo Pilligua;

Patricio Alfredo Vallejo Valdivieso

psicopedagogía se trabaje, además, con la prevención de abuso sexual infantil, incentivando a los padres a tener mayor pertinencia, por cuanto, un niño o niña abusada, a juicio de (de-Manuel-Vicente, 2017), presenta afecciones en los siguientes indicadores:

a) Cognitivos: retrasos en el desarrollo, retraso en el aprendizaje, déficit atencional.

b) Físicos: dificultad para caminar y sentarse; alteración en área anogenital como dolores, picores, hemorragias, desgarros, hematomas; infecciones genitales y del tracto urinario; enfermedades de transmisión sexual; embarazo.

c) Conductuales: el menor presenta conductas sexuales tales como masturbación compulsiva, conductas sexualizadas con otros niños, conocimientos sobre sexualidad que no son frecuentes a esa edad, negativa a ir con una determinada persona, cambios bruscos de conducta. Un indicador muy poderoso de abuso es el testimonio del niño, es decir, momento que el menor relata lo que le ha ocurrido, porque cuando un niño dice que ha sido víctima de abuso no miente casi nunca. Otra conducta que puede presentar es la retractación: el menor afirma que ha sido víctima de abuso y después lo niega. La retractación es un indicador de abuso. Los menores se retractan ante las amenazas y presiones del abusador, al comprobar el sufrimiento de su madre al enterarse o el peregrinaje al que se les somete de profesional en profesional.

d) Psicológicos: alteraciones del sueño y de la alimentación, enuresis, encopresis, depresión, conductas autolíticas, ansiedad, trastorno obsesivo-compulsivo, trastorno por estrés postraumático, sentimientos de culpa.

e) Sociales: aislamiento social, abuso de otros menores, desconfianza, consumo de tóxicos. 
La violencia sexual contra niños y niñas, no solo representa un abuso, sino, un delito, aunado a ser considerado en muchos casos como una patología por parte del victimario, de ese modo, se debe tener una perspectiva amplia para no encasillar al infante cuando presente problemas de atención, o de integración mediante una socialización asertiva, es necesario descubrir su historia de vida con la finalidad de iniciar un proceso de comprensión para ser incorporado a partir de ayuda psicológica - espiritual - afectiva, y desde una visión feminista en alteridad de la relación entre las partes involucradas, con la intención de ser abordadas desde una visión integral de los hechos (Fung-Fallas et al. 2020).

Otro aspecto a identificar por parte del personal de psicopedagogía, son los factores estresantes en el infante, a juicio de (Ferrara et al. 2016), esto es un indicador de posible presencia de abusos, siendo recurrente investigar para conocer el origen del estrés; lo cual, forma parte de una psicopedagogía incluyente donde se debe tener en consideración todos los elementos que se encuentran alrededor de la persona para ser incorporados al proceso pedagógico en ocasión de formar para una visión de respeto mutuo en conformidad a un crecimiento afectivo - sexual (Zambrano-Pincay et al. 2019), en concordancia con la valoración del otro como si fuese el yo, para lo cual es concerniente promover la alteridad en razón de la visión feminista del alter ego.

Para tal fin, se hace necesario modificar las estructuras mentales de los infantes para que se empoderen de un aprendizaje social con base a la alteridad y feminismo como variables generadoras de una visión significativa de la realidad a partir de iniciar un recorrido de experiencias educativas basadas en interiorizar a la otra persona (estudiantes con sus pares) con respeto, pero sin perder mi propia identidad, lo cual, en el tiempo pasa a la razón como un conocimiento significativo (Vallejo-Valdivieso et al. 2019).

Siendo transversal involucrar la ética feminista como un accionar para la educación de los infantes en razón de poder conciliar una ruta posible al crecimiento social del respeto 
al otro como un agente transformador de la realidad, en este sentido, (Tapia-González, 2017), introduce la obra de la feminista mexicana Graciela Hierro, para quien fue fundamental insistir en promover una pedagogía sustentada desde una óptica feminista en razón de promover nuevas estructuras no solo mentales, sino, sociales.

Para (Fernández-Guerrero, 2015), es fundamental abordar la educación en alteridad desde una óptica integral entre las cuales se encuentran la ética, lingüística, espiritual, como fundamentos esenciales para constituir un trabajo cooperativo entre los docentes y psicopedagogos, esto con la intención de propiciar estrategias con la finalidad de formar desde una óptica feminista en favor de propiciar un infante seguro de sí mismo en relación a profundizar en la conformación de un ciudadano con pensamiento critico global, siendo necesario además, inculcar la relación política en conformidad de proteger a la mujer de cualquier tipo de violencia (Castaño, 2021).

En cuanto al campo de la lingüística, se hace necesario enseñar desde los primeros años de formación académica, el discernimiento de los signos y códigos no feministas ocultos en la música, redes sociales, (Díaz-Altozano et al. 2021), con la intención de rechazar este tipo de arte y en consecuencia proceder a generar alternativas desde una generación que ha sido educada para respetar mutuamente, comprendiéndose que a través de los medios masivos y alternativos de comunicación, se ventila un fuerte medio para la degradación de la mujer, en especial cuando es sub valorada como objeto sexual, denominado por (Cobo-Bedia, 2015), como sobrecarga de la sexualidad.

\section{CONCLUSIÓN}

Al existir significancia bilateral de 0,046 para el momento de postest, se determina que el programa aplicado fue efectivo en la población que participó en la investigación. De ese modo, los docentes pueden diseñar estrategias desde la psicopedagogía para formar los estudiantes en respeto mutuo desde la perspectiva de género. 


\section{CIENCIAMATRIA}

Revista Interdisciplinaria de Humanidades, Educación, Ciencia y Tecnología

Año VII. Vol. VII. N¹. Edición Especial. 2021

Hecho el depósito de ley: pp201602FA4721

ISSN-L: 2542-3029; ISSN: 2610-802X

Universidad Nacional Experimental Francisco de Miranda (UNEFM). Santa Ana de Coro. Venezuela

Graciela Hernestina Zambrano-Pincay; María Jesús Viton-de-Antonio; Patricio Yosué Vallejo Pilligua;

Patricio Alfredo Vallejo Valdivieso

En cuanto a las madres, padres, en el rol de representantes que participaron en el programa de formación, cuentan ahora con la información necesaria para educar a sus hijos bajo una óptica de respeto mutuo desde la perspectiva de género, como una alternativa para educar desde el hogar a sus hijos o representados en favor de comprender la importancia que tiene el aceptarse así mismo y aceptar a las demás personas a partir de una relación asertiva desde el punto de vista emocional, evitándose así, situaciones de bullying social o escolar, además que pueden cooperar con los docentes en trabajar sobre un enfoque educativo basado en valores desde la perspectiva de género.

En este sentido, la psicopedagogía para educar desde una perspectiva de alteridad y feminismo, se enfrenta a grandes retos entre los cuales se encuentran la industria del arte musical, cinematográfico, el cual transmite una subvaloración de la mujer, viéndose por medios masivos, redes sociales, como sumisas en consideración de que deben prestar su cuerpo para posar como mercancía subvalorada por la sociedad, en este sentido, se debe formar para respetar al otro, comprenderlo, sin dejar la identidad propia de lado, sino, en un marco de alteridad para encontrar puntos de encuentros entre pares sociales.

\section{REFERENCIAS}

Castaño, P. (2021). Combatir la violencia contra las mujeres políticas. Investigaciones Feministas, 12(2), 623-637. https://doi.org/10.5209/infe.72868

Cobo-Bedia, R. (2015). El cuerpo de las mujeres y la sobrecarga de sexualidad. Investigaciones https://doi.org/10.5209/rev INFE.2015.v6.51376

de-Manuel-Vicente, C. (2017). Detectando el abuso sexual infantil. Pediatría Atención Primaria, 19(Supl. 26), 39-47. 
Díaz-Altozano, P., Padilla Castillo, G., \& Requeijo Rey, P. (2021). Sexualización de niñas en redes sociales: la necesidad de inteligencia semántica en Instagram. Investigaciones $\quad$ Feministas, 12(1), 31-45. https://doi.org/10.5209/infe.69559

Fernández-Guerrero, O. (2015). Levinas y la alteridad: cinco planos. Brocar. Cuadernos de Investigación Histórica, 0(39), 423-443. https://doi.org/10.18172/brocar.2902

Ferrara, P., Guadagno, C., Sbordone, A., Amato, M., Spina, G., Perrone, G., Cutrona, C., Basile, M. C., Ianniello, F., Fabrizio, G. C., Pettoello-Mantovani, M., Verrotti, A., Villani, A., \& Corsello, G. (2016). Child Abuse and Neglect and its Psycho-Physical and Social Consequences: A Review of the Literature. Current pediatric reviews, 12(4), 301-310. https://doi.org/10.2174/1573396312666160914193357

Fung-Fallas, M. P., Medina Correas, N. V., \& Quesada Musa, C. (2020). Abordaje del abuso sexual infantil. Revista Médica Sinergia, 5(4), e368. https://doi.org/10.31434/rms.v5i4.368

García-Piña, C. (2016). Sexualidad infantil: información para orientar la práctica clínica. Acta pediátrica de México, 37(1), 47-53.

Lozano-Chaguay, S, Robledos Galeas, R, \& Robledos Gáleas, S. (2020). La neuropsicología como referentes necesarios para comprender el comportamiento humano. Conrado, 16(73), 201-206.

Marañón, I. (2017). Educar en el feminismo. Plataforma editorial. Barcelona, España.

Ortega, P. y otros (2014). Educar en la Alteridad [Educate in Alterity]. Libro primero del tomo I, Colección Pedagogía de la Alteridad. Colombia: Redipe y Editum. https://hdl.handle.net/10171/37404

Pérez, D. (2015). La orientación psicopedagógica con perspectiva de género. Revista Ágora Trujillo, 18(36).

Tapia-González, G. (2017). Graciela Hierro: Filosofía de la educación en clave de género. Revista interdisciplinaria de estudios de género de El Colegio de México, 3(5), 1-22. https://doi.org/10.24201/eg.v3i5.94 


\section{CIENCIAMATRIA}

Revista Interdisciplinaria de Humanidades, Educación, Ciencia y Tecnología

Año VII. Vol. VII. N¹. Edición Especial. 2021

Hecho el depósito de ley: pp201602FA4721

ISSN-L: 2542-3029; ISSN: 2610-802X

Universidad Nacional Experimental Francisco de Miranda (UNEFM). Santa Ana de Coro. Venezuela

Graciela Hernestina Zambrano-Pincay; María Jesús Viton-de-Antonio; Patricio Yosué Vallejo Pilligua;

Patricio Alfredo Vallejo Valdivieso

Urruzola-Zabalza, M. (2000). La educación de las relaciones afectivas y sexuales. http://hdl.handle.net/11162/36631

Vallejo-Valdivieso, P., Zambrano Pincay, G., Vallejo Pilligua, P., \& Bravo Cedeño, G. (2019). Estructuras mentales en la construcción de aprendizaje significativo. CIENCIAMATRIA, 5(8), 228-241. https://doi.org/10.35381/cm.v5i8.257

Zambrano-Pincay, G., Vallejo Valdivieso, P., Vallejo Pilligua, P., \& Bravo Cedeño, G. (2019). Los profesionales de la Psicopedagogía en la atención a la diversidad como Agente Educativo. Revista Arbitrada Interdisciplinaria de Ciencias de la Salud. Salud y Vida, 3(6), 41-57. http://dx.doi.org/10.35381/s.v.v3i6.304

Zanon, A. (2019). El principio de la alteridad de Lévinas como fundamento para la responsabilidad ética. Perseitas, $8, \quad 75 \quad$ - 103. https://doi.org/10.21501/23461780.3489 